\title{
CONNECTEDNESS IN SYNTOPOGENOUS SPACES
}

\author{
J. L. SIEBER AND W. J. PERVIN ${ }^{1}$
}

Introduction. In his monograph [1], Császár introduced the notion of a syntopogenous space, which generalized the notions of a topological space, a proximity space, and a uniform space. Although Császár was able to obtain many of the usual theorems of general topology in this more general setting, the basic topological notion of connectedness was not introduced at all. Mrówka and Pervin [2] have discussed the concepts of $\mathcal{u}$-connectedness and $\delta$-connectedness for uniform and proximity spaces, respectively. In this paper we shall give a definition for connectedness in syntopogenous spaces and show that it agrees with the corresponding properties in topological, proximity, and uniform spaces. Furthermore, we shall obtain some of the theorems concerning connected sets from general topology in this more general setting. Throughout, we shall use the notation due to Császár and assume a familiarity with his monograph [1].

1. Definitions and elementary properties. Let $[E, \delta]$ be an arbitrary syntopogenous space. Two subsets $A$ and $B$ of $E$ will be called S-separated iff $A<E-B$ and $B<E-A$ for some $<\in \mathcal{S}$. The space $[E, \delta]$ will be said to be $\delta$-connected iff it is not the union of two nonempty $S$-separated subsets. This is clearly equivalent to the requirement that there is no nonempty proper subset $A$ of $E$ such that $A<A$ and $E-A<E-A$ for some $<\in S$.

THEOREM 1.1. [E, $s]$ is $S$-connected iff every (S, D)-continuous mapping of $E$ into a discrete space $[D, D]$ is constant.

Proof. Suppose $f$ is an (S, D)-continuous mapping of $E$ into the discrete space $[D, D]$, and suppose $p \in f(E)$. Since $D=\{\subset\}$ is the discrete structure, $p<p$ since $\{p\} \subset\{p\}$ and $D-\{p\}<D-\{p\}$ since $D-\{p\} \subset D-\{p\}$. Since $f$ is $(\mathcal{S}, D)$-continuous, there must exist some $<^{\prime} \in \mathcal{S}$ such that $f^{-1}(p)<^{\prime} f^{-1}(p)$ and $E-f^{-1}(p)<^{\prime} E-f^{-1}(p)$. Thus if $f$ was not constant, $f^{-1}(p)$ would be a nonempty proper subset, and thus $[E, \mathcal{S}]$ would not be $\delta$-connected. Conversely, if $A$ and $B$ are $\mathcal{S}$-separated subsets whose union is $E$, the mapping $f$ of $E$ into the twopoint discrete space $[\{a, b\}, C]$ defined by setting $f(A)=a$ and $f(B)=b$ is $(S, D)$-continuous. If $A$ and $B$ are nonempty, it will be nonconstant.

\footnotetext{
Received by the editors April 22, 1963.

1 The authors were supported by the National Science Foundation under research grant NSF G-22690.
} 
We note that $[D, D]$ could always be taken to be a two-point discrete space with no loss of generality. For symmetric spaces $[E, s]$, $S$-connectedness is equivalent to the requirement that there is no nonempty proper subset $A \subset E$ such that $A<A$ for some $<\in \mathcal{S}$.

Corollary 1.2. If $f$ is an $\left(s, s^{\prime}\right)$-continuous mapping of $[E, s]$ onto $\left[E^{\prime}, \mathcal{S}^{\prime}\right]$ and $[E, \delta]$ is $\mathcal{S}$-connected, then $\left[E^{\prime}, \mathrm{s}^{\prime}\right]$ is $\mathrm{s}^{\prime}$-connected.

A subset $A$ of $[E, \S]$ will be called $\delta$-connected iff $[A, \delta \mid A]$ is $\S \mid A$-connected (see [1, p. 94]). It follows immediately that $A$ is $\delta$-connected iff it is not the union of two nonempty $\mathcal{S}$-separated subsets.

TheOREm 1.3. If $A$ is an $s$-connected subset of $[E, \delta]$ and $A \subset M \cup N$ where $M$ and $N$ are $S$-separated subsets of $E$, then either $A \subset M$ or $A \subset N$.

Proof. By Axiom $\left(O_{3}\right)$ of $[1$, p. 21], $A \cap M$ and $A \cap N$ will be $S$-separated subsets of $A$ whose union will be $A$. If $A$ is $S$-connected, one of these sets must be empty and so the conclusion follows.

Corollary 1.4. If $A$ and $B$ are two s-connected subsets of $[E, s]$ which are not $S$-separated, then $A \cup B$ is s-connected.

Proof. If we write $A \cup B=M \cup N$ where $M$ and $N$ are $S$-separated subsets of $E$, then, by the previous theorem, either $A$ and $B$ are $\delta$ separated or else one of the subsets $M$ and $N$ must be empty.

Corollary 1.5. If $A$ is an s-connected subset of $[E, \delta]$ and $B \subset E$ is such that $A \subset B \subset \bar{A}$, then $B$ is s-connected.

Proof. We note that the closure of $A$, denoted $\bar{A}$, is $\{p \in E: p<M$ for some $<\in S$ and $M \subset E$ implies $M \cap A \neq \varnothing\}$; that is, those points $p$ for which every neighborhood intersects $A$. If we write $B$ as the union of two $S$-separated subsets $M$ and $N$, then, by the previous theorem, either $A \subset M$ or $A \subset N$. Let us assume that $A \subset M$. Now if $p \in N$, then $p \in B$ and, since $M$ and $N$ are $S$-separated, $p<E-M$ $C E-A$ which implies that $p<E-A$ for some $<\in s$ by Axiom $\left(O_{3}\right)$. Now $p \in \bar{A}$ and $p<E-A$ imply that $(E-A) \cap A \neq \varnothing$, which is a contradiction. Hence $N$ must be empty and so $B$ is $\delta$-connected.

Corollary 1.6. If $\left\{A_{\lambda}: \lambda \in \Lambda\right\}$ is a family of s-connected subsets of $[E, \S]$, and $A_{\lambda_{0}}\left(\lambda_{0} \in \Lambda\right)$ is not $\delta$-separated from any $A_{\lambda}$, then $A=\bigcup_{\lambda \in \Delta} A_{\lambda}$ is S-connected.

Proof. This follows immediately from the above theorem.

In particular, if $\bigcap_{\lambda \in \Delta} A_{\lambda} \neq \varnothing$ in the last corollary, then $A$ is $\mathrm{S}$ - 
connected. From this it follows that the union of all $s$-connected subsets of $E$ which contain a fixed point $p \in E$ is an $S$-connected set which we will call the $S$-component of $p$, denoted by $C_{\mathcal{S}}(p)$. It is clearly the maximal $s$-connected set containing $p$.

By Corollary 1.4 , if $C_{\mathcal{S}}(p)$ and $C_{\mathcal{S}}(q)$ are not $\delta$-separated, then their union must be $\delta$-connected, and by the maximality of components, we must have $C_{\mathcal{S}}(p)=C_{\mathcal{S}}(q)$. Thus a syntopogenous space $[E, \delta]$ is divided up into disjoint, $\delta$-separated $\delta$-components. Clearly, by Corollary 1.5 , these components are closed sets.

A final elementary theorem with a topological analogy concerns the product of connected spaces. If $\left\{\left[E^{\lambda}, s^{\lambda}\right]\right\}_{\lambda \in \Lambda}$ is a nonempty family of nonempty syntopogenous spaces, then their product space $[E, \delta]$ where $E=X_{\lambda \in \Lambda} E^{\lambda}$ and $\delta=X_{\lambda \in \Lambda} \delta^{\lambda}$ has been defined (see [1, p. 115]).

TheOREM 1.7. The product space $[E, \delta]$ is $\delta$-connected iff each space $\left[E^{\lambda}, \mathcal{S}^{\lambda}\right]$ is $S^{\lambda}$-connected.

Proof. Since the projections are $\left(\delta, \delta^{\lambda}\right)$-continuous, if $[E, \delta]$ is $\delta$-connected, then each $\left[E^{\lambda}, \delta^{\lambda}\right]$ is $\delta^{\lambda}$-connected by Corollary 1.2. Conversely, suppose each $\left[E^{\lambda}, S^{\lambda}\right]$ is $\delta^{\lambda}$-connected. Let $\left\{z^{\lambda}\right\}$ be a fixed point of $X_{\lambda \in \Lambda} E^{\lambda}$. By (11.29) of $\left[1\right.$, p. 122], the subset $E_{\beta}$ of the product space consisting of all points $\left\{x^{\lambda}\right\}$ such that $x^{\lambda}=z^{\lambda}$ if $\lambda \neq \beta$ while $x^{\beta}$ may be any point of $E^{\beta}$ is isomorphic to $E^{\beta}$, and hence is an $\delta$-connected set containing $\left\{z^{\lambda}\right\}$. Clearly, for any finite number of indices $\beta_{1}, \beta_{2}, \cdots, \beta_{m}, E_{\beta_{1}} \times E_{\beta_{2}} \times \cdots \times E_{\beta_{m}}$, that is, the set of all points $\left\{x^{\lambda}\right\}$ such that $x^{\lambda}=z^{\lambda}$ if $\lambda \neq \beta_{1}, \beta_{2}, \cdots, \beta_{m}$, is $S$-connected and contains $\left\{z^{\lambda}\right\}$. Since the $S$-neighborhoods of points include isomorphic copies of all but a finite number of the coordinate spaces (see (11.14) of $[1$, p. 117]), sets of this form are dense in $E$ and so, by Corollary $1.5,[E, \delta]$ is $S$-connected.

2. Real functions and applications. It is immediate from the definition that $\delta$-connectivity is equivalent to $S^{c}$-connectivity and $S^{t}$-connectivity (see (8.5) and (8.30) of $[1$, pp. 73, 76]). Furthermore, if $S$ is coarser than $\mathcal{S}^{\prime}$ and a space is $\mathcal{S}^{\prime}$-connected, then it is clearly $\mathcal{S}$-connected (see $(10.10)$ of $[1$, p. 105]). From this it follows that if a space is $\mathcal{S}^{s}$-, $\mathcal{S}^{b}$-, or $\mathcal{S}^{p}$-connected, then it is $\mathcal{S}$-connected.

TheOREM 2.1. If $[E, \mathcal{S}]$ is $\mathcal{S}$-connected and compact, then it is $\mathcal{S}^{t p_{-}}$ connected.

Proof. Let $f$ be any $\left(\delta^{t p}, D\right)$-continuous mapping of $E$ into a discrete space $[D, D]$. Since $\delta$ is compact, $\mathcal{S}^{t}$ is compact by $(15.49)$ of 
$\left[1\right.$, p. 197] so $f$ is $\left(S^{t}, D\right)$-continuous by $(15.57)$ of $[1$, p. 198]. Since $E$ is $S$-connected, it is $S^{t}$-connected and therefore $f$ must be constant by Theorem 1.1 .

We note that this theorem generalizes the property of uniform spaces which states that a compact uniform space is uniformly connected iff it is connected.

Let us turn now to a characterization of $s$-connectedness in terms of real-valued functions. As is well known, the connected (i.e., $\mathcal{F}^{t p_{-}}$ connected in the notation of $[1$, p. 81]) subsets of the real line are the intervals (in which statement we will include the degenerate cases of the single point sets and the empty set). As was shown by Mrówka and Pervin [2], the equiconnected and uniformly connected subsets (i.e., $\mathfrak{F}^{t}$ - and $\mathfrak{F}^{\mathcal{C}}$-connected in $[1$, p. 80$]$ ) are those sets which are dense in an interval.

Lemma 2.2. A subset of the real line is $g^{8}$-connected (see $\left.[1, p .79]\right)$ iff it is dense in an interval.

Proof. A set which is dense in an interval is $\mathfrak{H}$-connected as mentioned above, hence, since $g^{b b}=\mathfrak{H C}$ by definition, it is $\mathscr{g}^{8}$-connected by the discussion at the beginning of this section. Now suppose $A$ is a subset of the real line which is not dense in an interval. Then there exists a point $z$ and an $\epsilon>0$ such that $A \cap(z-\epsilon, z+\epsilon)=\varnothing$ but $A \cap(-\infty, z) \neq \varnothing \neq A \cap(z,+\infty)$. We then have, in the notation of (2.3) of $[1$, p. 22],

$$
A \cap(-\infty, z-\epsilon]<, E-(A \cap[z+\epsilon,+\infty)) .
$$

Thus

$$
A \cap(-\infty, z-\epsilon]<E-(A \cap[z+\epsilon,+\infty))
$$

and since $g^{*}$ is symmetric, the sets $A \cap(-\infty, z-\epsilon]$ and $A \cap[z+\epsilon,+\infty)$ are $g^{s}$-separated. Thus $A$ is not $g^{s}$-connected.

It is interesting to note that every subset of the real line is $g$ connected (see [1, p. 63]). The above characterizations of various connectivities for real sets leads to the following result.

Theorem 2.3. A syntopogenous space $[E, s]$ is s-connected iff every real-valued $\left(\mathcal{S}, \mathcal{S}^{\prime}\right)$-continuous mapping has a range which is $\boldsymbol{S}^{\prime}$-connected, where $\mathcal{S}^{\prime}$ may be $\mathcal{G}^{\star}, g^{\star b}=\mathfrak{H C}$, $\mathfrak{F C}^{t}$, or $\mathfrak{F C}^{t p}$.

Proof. By Corollary 1.2 , if $[E, \delta]$ is $S$-connected, then its image under an $\left(\boldsymbol{S}, \boldsymbol{S}^{\prime}\right)$-continuous mapping is certainly $\mathfrak{S}^{\prime}$-connected. Now suppose $[E, \S]$ is not $\$$-connected and let $A$ and $B$ be two $\delta$-separated 
subsets whose union is $E$. Thus we have $A<E-B$ and $B<E-A$ for some $<\in S$. If we define a mapping $f$ of $E$ into the subset $\{0,1\}$ of the reals by setting $f(p)=0$ if $p \in A$ and $f(p)=1$ if $p \in B$, then the range of this function is not $\delta^{\prime}$-connected for $S^{\prime}$ equal to $\mathscr{S}^{*}, \mathfrak{H}^{\mathcal{C}}, \mathfrak{F}^{t}$, or $\mathfrak{F}^{t p}$. However, $f$ is $\left(s, s^{\prime}\right)$-continuous since each of these structures restricted to the set $\{0,1\}$ is just the discrete structure.

It is clear from Theorem 1.1 that our definition of $S$-connectedness agrees in the case of uniform and proximity spaces with the usual definition which is that every uniformly continuous or equicontinuous mapping into a discrete space is constant. In particular, since a quasiuniform space $[E, \mathcal{u}]$ is associated in a one-to-one fashion to some biperfect syntopogenous space $[E, \varsigma],[E, \mathcal{U}]$ is $\mathcal{u}$-connected iff $[E, \S]$ is $\mathcal{S}$-connected. Hence, two subsets $A$ and $B$ of $E$ are $\mathcal{U}$-separated iff $U[A] \cap B=A \cap U[B]=\varnothing$ for some $U \in \mathcal{u}$, and $[E, \mathfrak{u}]$ is $\mathfrak{u}$-connected iff it is not the union of two nonempty $\mathcal{U}$-separated subsets. For a uniform space $[E, \mathcal{u}], A$ and $B$ are $\mathcal{u}$-separated iff $U[A] \cap U[B]$ $=\varnothing$ for some $U \in \mathcal{u}$. On the other hand, each proximity space $[E, \delta]$ is associated with a unique simple symmetric syntopogenous space $[E, \delta]$, and $[E, \delta]$ is $\delta$-connected iff $[E, \delta]$ is $\delta$-connected. Hence, two subsets $A, B \subset E$ are $\delta$-separated iff $A \bar{\delta} B$, and $[E, \delta]$ is $\delta$-connected iff it is not the union of two nonempty $\delta$-separated subsets.

In the case where $S$ is a simple perfect syntopogenous structure and so associated with a classical topology, the above theorem gives the well-known result that a topological space is connected iff every continuous real-valued function has the Darboux property, where we use $\mathcal{S}^{\prime}=\mathfrak{F C}^{t p}$ the simple perfect structure associated with the usual topology for the reals. If $S$ is a symmetric perfect (symmetric simple) syntopogenous structure and so associated with a uniformity (proximity) for $E$, then the above theorem gives the result of Mrówka and Pervin that a uniform (proximity) space is $\mathcal{u}$-connected ( $\delta$ connected) iff the range of every real-valued uniformly continuous (equicontinuous) mapping of $E$ is dense in an interval, where we use $\mathcal{S}^{\prime}=\mathfrak{F C}\left(\mathcal{S}^{\prime}=\mathfrak{H C}^{t}\right)$ the symmetric perfect (symmetric simple) syntopogenous structure associated with the usual uniformity (proximity) for the reals.

From the remarks at the beginning of this section, we see that every connected topological space is $u$-connected and $\delta$-connected with respect to every uniformity $\mathcal{U}$ and proximity $\delta$ which generates that topology. Let $[E, g]$ be a Tychonoff space (i.e., completely regular and $T_{1}$ ) and let $\delta_{\beta}$ be the proximity on $E$ which is associated with the Stone-Cech compactification $\beta E$ of $E$; i.e., $A \delta_{\beta} B$ iff $c_{\beta}(A) \cap c_{\beta}(B)$ $\neq \varnothing$ where $c_{\beta}$ denotes the closure operator in $\beta E$. If $f$ is a continuous 
map of $[E, g]$ into a discrete space, then $f$ has a continuous extension $f^{*}$ on $\beta E$ which must be equicontinuous since $\beta E$ is compact. Hence the restriction $f^{*} \mid E=f$ must be equicontinuous with respect to $\delta_{\beta}$. Thus a Tychonoff space is connected iff it is $\delta_{\beta}$-connected.

Finally, in regard to Theorem 1.7, it should be noted that the product space $[E, \delta]$ of syntopogenous spaces $\left[E^{\lambda}, \delta^{\lambda}\right]$ may not be simple or perfect even if each $\delta^{\lambda}$ has the property. The usual products of quasi-uniform and proximity spaces are obtained in a canonical way by Császár [1, pp. 122-126] by considering $\delta^{b}$ and $\delta^{t}$, respectively.

Theorem 2.4. The product of U-connected ( $\delta$-connected) spaces is

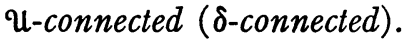

Proof. For $\delta$-connectedness, the result follows from the fact that $\boldsymbol{S}$ - and $\boldsymbol{S}^{t}$-connectedness are equivalent. Now suppose each space $\left[E^{\lambda}, \mathcal{U}^{\lambda}\right](\lambda \in \Lambda)$ is $\mathcal{U}^{\lambda}$-connected. The sets considered in the proof of Theorem 1.7 are dense in $E$ not only with respect to $\delta=X_{\lambda \in \Delta} \delta^{\lambda}$ (where $\delta^{\lambda}$ is the biperfect syntopology associated with $\mathcal{u}^{\lambda}$ ) but also with respect to $\delta^{b}$. This follows from the formula $\delta^{b t p}=\left(X_{\lambda \in \Lambda} \delta^{\lambda t p}\right)^{t p}$ $=\left(X_{\lambda \in \Delta} S^{\lambda}\right)^{t p}$ (see $(11.26)$ and $(11.25)$ of $[1$, pp. 121, 120]) since a set is dense with respect to $S$ iff it is dense with respect to $\mathcal{S}^{t p}$. Furthermore, the isomorphism of $\left[E^{\beta}, \delta^{\beta}\right]$ onto $\left[E_{\beta}, s \mid E_{\beta}\right]$ yields an isomorphism of $\left[E^{\beta}, \mathcal{S}^{\beta}\right]$ onto $\left[E_{\beta},\left(\mathcal{S} \mid E_{\beta}\right)^{b}\right]$ since $\mathcal{S}^{\beta b}=\mathcal{S}^{\beta}$. By $(9.11)$ of $[1$, p. 94], $\left(\delta \mid E_{\beta}\right)^{b}=\varsigma^{b} \mid E_{\beta}$ so the sets considered in the proof of Theorem 1.7 are also $\delta^{b}$-connected.

\section{REFERENCES}

1. Åkos Császár, Fondements de la topologie générale, Gauthier-Villars, Paris, 1960.

2. S. G. Mrówka and W. J. Pervin, On uniform connectedness, Proc. Amer. Math. Soc. 15 (1964), 446-449.

Pennsylvania State University 